\title{
Self-Cleaning Barrier Filter for Rotorcraft Engines
}

\author{
N. Bojdo ${ }^{\mathrm{a}, 1, *}$, A. Filippone $\mathrm{a}^{\mathrm{a}, 2}$ \\ ${ }^{a}$ Department of Mechanical, Aerospace $\mathcal{E}$ Civil Engineering, George Begg Building, \\ University of Manchester, Manchester, United Kingdom, M13 9PL
}

\begin{abstract}
A parameter-based study of a novel self-cleaning barrier filter concept for rotorcraft engines is presented. Existing engie inlet barrier filters for rotorcraft achieve a high separation efficiency (up to $99 \%$ for a dust resembling $\mathrm{AC}$ Coarse test dust) at the expense of a pressure drop that increases over time. Other engine protection devices with invariant pressure loss signals cannot offer the same level of efficiency. Furthermore, inlet barrier filters are absent on rotocraft with larger mas flow demands, due to larger filtration area required. This work proposes a solution that addresses each of these limitations, and establishes the parametric framework upon which such a design could be realised. The drum-shaped self-cleaning barrier filter fits to the engine inlet and spins about its longitudinal axis, such that each of its fibrous filter pleats can be cleaned once per cycle. An initial "ramping up" of pressure loss gives way to a period of sustained invariance. The key parameters are the internal particle capacity of the chosen filter medium and the speed of rotation. Other design parameters can be optimised to yield
\end{abstract}

\footnotetext{
* Corresponding author.

Email addresses: nicholas.bojdo@postgrad.manchester.ac.uk (N. Bojdo), a.filippone@manchester.ac.uk (A. Filippone)

${ }^{1}$ Postdoctoral Researcher.

${ }^{2}$ Senior Lecturer.
} 
minimum pressure drop for given set of flow conditions.

Keywords: Inlet Barrier Filter, Self-cleaning Pleated Filter, EAPS, Helicopter Engine Protection, Turboshaft Engine

\section{Nomenclature}

Acronyms

$A C=$ Air Cleaner
$A P C D=$ Air Pollution Control Device
$C F D=$ Computational Fluid Dynamics
$E A P S=$ Engine Air Particle Separator
$H E P A=$ High Efficiency Particulate Air
$I B F=$ Inlet Barrier Filter
$I C A O=$ International Civil Aviation Organization
$I P S=$ Integrated Inertial Particle Separators
$I S A$
$M T O W=$ International Standard Atmosphere
$P J A C=$ Pulse-Jet Air Cleaner
$P S D=$ Particulate Size Distribution
$R P M=$ Revolutions Per Minute
$S A E=$ Society of Automotive Engineers




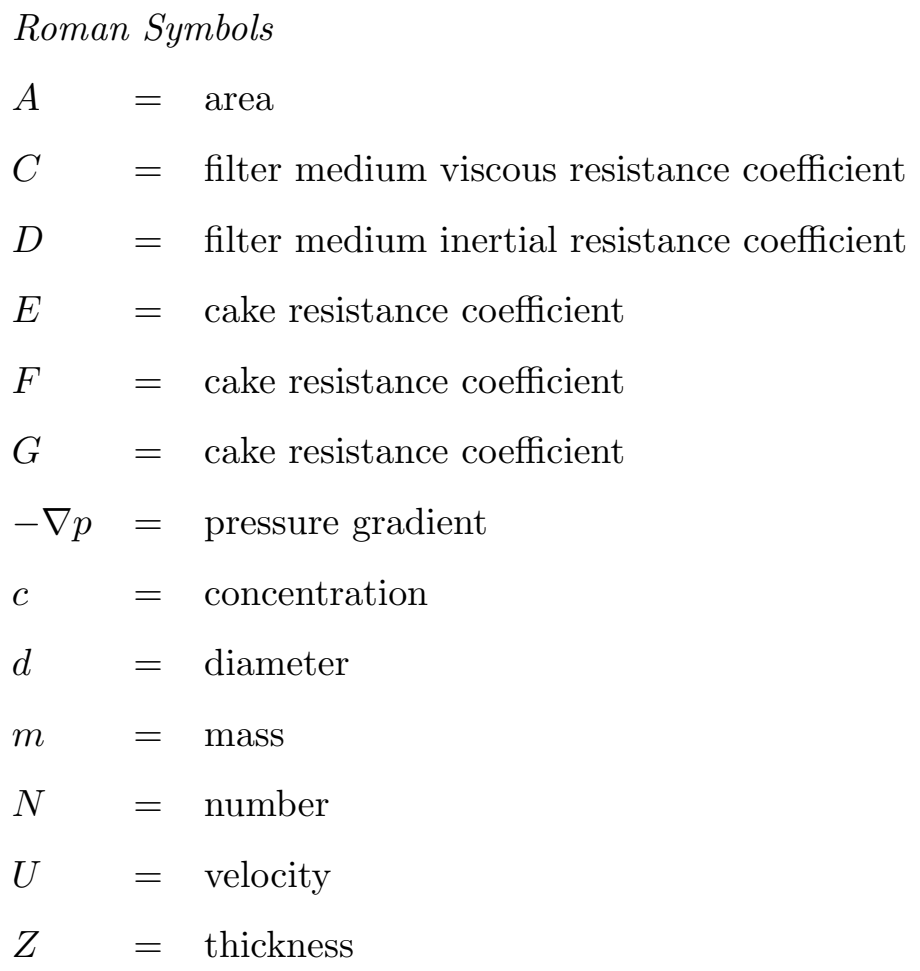

Greek Symbols

$\epsilon=$ porosity

$\kappa=$ particle dynamic shape factor

$\mu=$ kinematic viscosity

$\rho=$ density

$\sigma=$ standard deviation

$v=$ cake void function 


$$
\begin{aligned}
& \text { Subscripts } \\
& {[\cdot]_{C}=\text { of cake }} \\
& {[\cdot]_{f}=\text { of fibre }} \\
& {[\cdot]_{F}=\text { of filter }} \\
& {[\cdot]_{g}=\text { of gas }} \\
& {[\cdot]_{g}=\text { of geometric }} \\
& {[\cdot]_{m}=\text { of mass }} \\
& {[\cdot]_{p}=\text { of particulate }} \\
& {[\cdot]_{p c}=\text { of collected particulate }} \\
& {[\cdot]_{v}=\text { of volume }} \\
& \text { Superscripts } \\
& {[.]^{+} \quad=\quad \text { dimensionless }}
\end{aligned}
$$

\section{Introduction}

Pleated filters for air filtration are found in many applications, most notably in the automotive industry. This contribution concerns their use in cleaning helicopter engine inflow air. Pleated filter media offer several benefits over their planar counterparts, such as increased rigidity. However, it is the increase filtration surface area per unit projected area that motivates their implementation, permitting their use in filtering contaminated gas at high flow rates (of the order $10^{-1}$ to $10^{0} \mathrm{~m}^{-3} \mathrm{~s}^{-1} \mathrm{~m}^{-2}$ ). The term Inlet Barrier Filter (IBF) is appropriated to one such embodiment of a pleated filter, found integrated into the air intake of a helicopter engine when dusty conditions prevail. It competes with other so-called Engine Air Particle Separation (EAPS) technologies, as discussed in Chapter 7 of Bojdo [1], 
to prevent the ingestion of potentially damaging particles with a minimum loss of pressure and engine air mass flow rate. A typical IBF installation is shown in Figure 1 attached to the intake of a medium-lift helicopter.

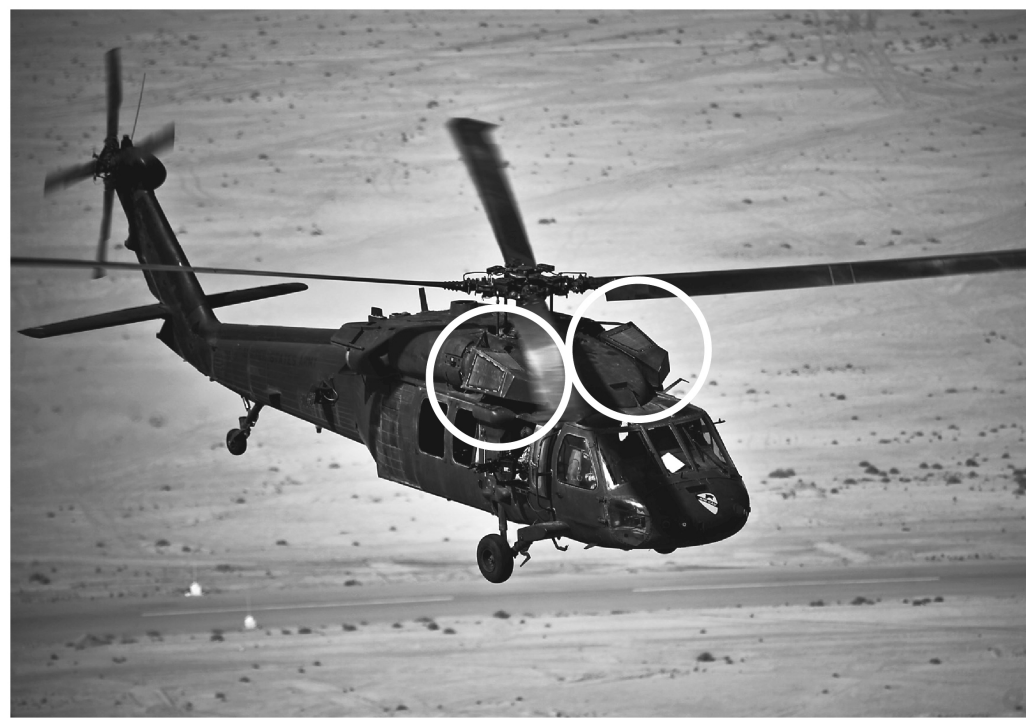

Figure 1: A typical IBF, circled, attached to the intake of a medium-lift helicopter.

The IBF performs well in this duty, for example achieving a mass removal rate of $99 \%$ when subjected to AC Coarse test dust. However, as with all traditional porous media-based filters, the performance of an IBF deteriorates over time, due to the accumulation of particles. This demands constant monitoring and regular cleaning of the filter panel, to ensure the increasing pneumatic resistance does not reach an unreasonable level. For the helicopter operator, the consequent transiency of the inlet condition coupled with the inconvenience of removing and cleaning the filters undermines the benefit of protection offered by the IBF. If, however, the IBF were made to self-clean, such obstacles to outright success could be overcome. It is the 
objective of the current work to investigate such a concept.

\section{Background}

The concept of the self-cleaning filter is nothing new. A wealth of technology has developed around dust collection and removal, not only in attempts to improve filter performance, but also in allowing valuable particles to be retained. Generally speaking, there are three methods of particle removal once a cake has developed on a fabric filter surface: shaking, reverse-air, and pulse-jet cleaning. Such methods are commonplace in large industrial "baghouse cleaners" - an air pollution control device (APCD) containing many cylindrical, upright filter bags that are inexpensive, easy-to-use, highly efficient at collecting particles, and consequently most widespread $[2,3,4]$. "Pulse-jet" is the most commonly applied method, whereby a short duration (50-150 ms) jet of high-pressure (3-7 bar) air is injected in the reverse-flow direction, causing a sudden expansion of the filter medium and a dislodging of the surface cake, which drops into a hopper below [2]. Crucially, the impulse must be strong enough to overcome the adhesive force between the cake and the filter or the cohesive force inside the dust cake. Lo et al. $[3,5]$ revealed that the efficiency of cleaning is also dependent on the pleat geometry (height to pitch ratio), distance from the pulse-jet nozzle, the medium surface finish, medium permeability and the chosen cleaning schedule (at regular time intervals or "on demand"). Such a multitude of parameters renders the academic field more conducive to casespecific optimisation studies, such as that offered by Park et al. [6], in which the optimum pleat height to pitch ratio was found to be 1.48 , above which filter cleaning became difficult.

While baghouse filter research predominantly focuses on optimisation 
with respect to dust collection efficiency and residual pressure drop, other studies investigate pleated filters of low dust loading, such as HEPA filters. In such cases, the critical parameters are the initial pressure drop and collection efficiency; the formation of a cake may occur, but its presence is not often a determining factor in the optimum design. Modelling focuses at the macro scale. Fotovati et al. [16] recently developed user-defined sub-routines to numerically model the instantaneous collection efficiency and pressure drop of pleated filters based particle deposition within the filter, using a commercially available computational fluid dynamics (CFD) code. The inlet velocities sampled reached a maximum of $0.5 \mathrm{~ms}^{-1}$, and filter clogging was modelled in two dimensions throughout the filter medium depth. A similar, less computationally expensive one-dimensional pleated filter clogging model was developed by Rebaï et al. [8] for flows of an order of magnitude higher, assuming laminar flow and a particle deposition rate proportional to the local filtration velocity. In both studies, an optimum design point was discussed, at which a minimum initial pressure drop or a maximum holding capacity could be achieved by setting an optimum pleat geometry, the former being an idea first identified by Chen et al. [7].

With respect to the field of filtration and separation, the aim of our study lies somewhere between the two applications. Receiving intake air with a particulate concentration of up to $2.5 \mathrm{gm}^{-3}$, at an engine mass flow rate of 5 $\mathrm{kgs}^{-1}$, a typical IBF collects around 0.6 kilograms of dust per minute. While such high concentrations are common in baghouse cleaners, the space in the intake plenum, in which an IBF must fit, is far less accommodating than the almost limitless area afforded to the former. Consequently, a typical superficial velocity (volume flow rate divided by projected filter area) is in the region of 3 to $10 \mathrm{~ms}^{-1}[9]$, which translates to filtration velocities of the 
order $1 \mathrm{~ms}^{-1}$. This is two orders of magnitude higher than the face velocities found in the studies by Lo et al. $[3,5]$, and an order of magnitude higher than range used in the work of Fotovati et al. [16]. Furthermore, in existing studies the flows are all assumed to be laminar; in the region directly beneath a helicopter rotor disk, the chaotic flow being drawn into the engine is likely to be turbulent. Hence the conditions in the current application of pleated filters are different to those that have been modelled in prior work.

With respect to the field of helicopter engine protection, there are currently no studies in the literature that predict the performance of an installed IBF using CFD or analytical theory of pleats of this size, other than those published by the authors (see Refs. $[9,10,11,12]$ ). Of work pertaining to IBF, there are two notable contributions. The first is a joint presentation by Scimone \& Frey et al. [14]. In this paper the authors detail the background, filter media technology, design considerations, and predict the effect on engine performance and lifetime increase using simulation programs. While providing useful insight into the state of the art, no allusion is made to the design particulars of the IBF and no real test results are given. Instead, the focus is on comparing the IBF technology to the other particle separators and describing the main design considerations of an IBF. Elsewhere in the literature is a contribution by Ockier et al. [15] in which the flight testing and certification of an IBF is given. Details of the instrumentation used during flight testing are provided. The most significant detail that can be inferred from this work is the temporal loss of pressure across the IBF, which was obtained during extensive operational evaluation in the Mojave Desert, California. Again, no actual data are provided but the pressure loss is shown to rise to a "warning level" after 12 minutes spent in a heavy dust cloud during takeoff and landing (equivalent to 30 landings in full brownout 
conditions, according to the authors). This level is thought to correspond to a pressure drop of around 3,000 $\mathrm{Pa}$. In prior work, the present authors aimed to infer as much technical information from the existing literature and commercial product descriptions on IBF to permit a theoretical study. The CFD results from that study are extrapolated for use in the current work.

In addition to the limitations described in the introduction, IBF are not found on large rotorcraft. This is thought to be due to the greater mass flow requirement of the engine, which would demand too large a projected filter area in order to maintain a reasonable filtration velocity. In lieu of an IBF or alternative retro-fit EAPS technology, such engines are fitted with Inlet Particle Separators which, unlike the IBF, fail to separate well particles below $10 \mu \mathrm{m}$ [13], which can still cause damage to engine components.

This paper aims to establish the design parameters for a new self-cleaning inlet barrier filter, that could cope with an equivalent mass flow rate while providing a high separation efficiency at the expense of a pressure loss that remains invariant.

\section{Methods}

It would be futile to attempt an optimisation study or to develop an intricate model of filter ageing, without experimental data. There are several challenges that must be addressed before such studies can be made. They are:

1. How can a self-cleaning model be integrated into an existing engine intake?

2. What are the relevant design parameters? 
3. Is the performance envelope realistic and achievable?

Hence our contribution lays out an initial design framework for a selfcleaning barrier filter for large helicopter engines. Some existing large helicopter engines are fitted with Integrated Inertial Particle Separators (IPS), which force the influent air around an obstruction in order to impart an outward radial component of velocity to the particulate-laden stream, causing separation by inertia. An external-type IPS device is shown in Figure 2. Internal-type IPS are common too; for a comprehensive description with illustrations of different embodiments, see Filippone \& Bojdo [10].

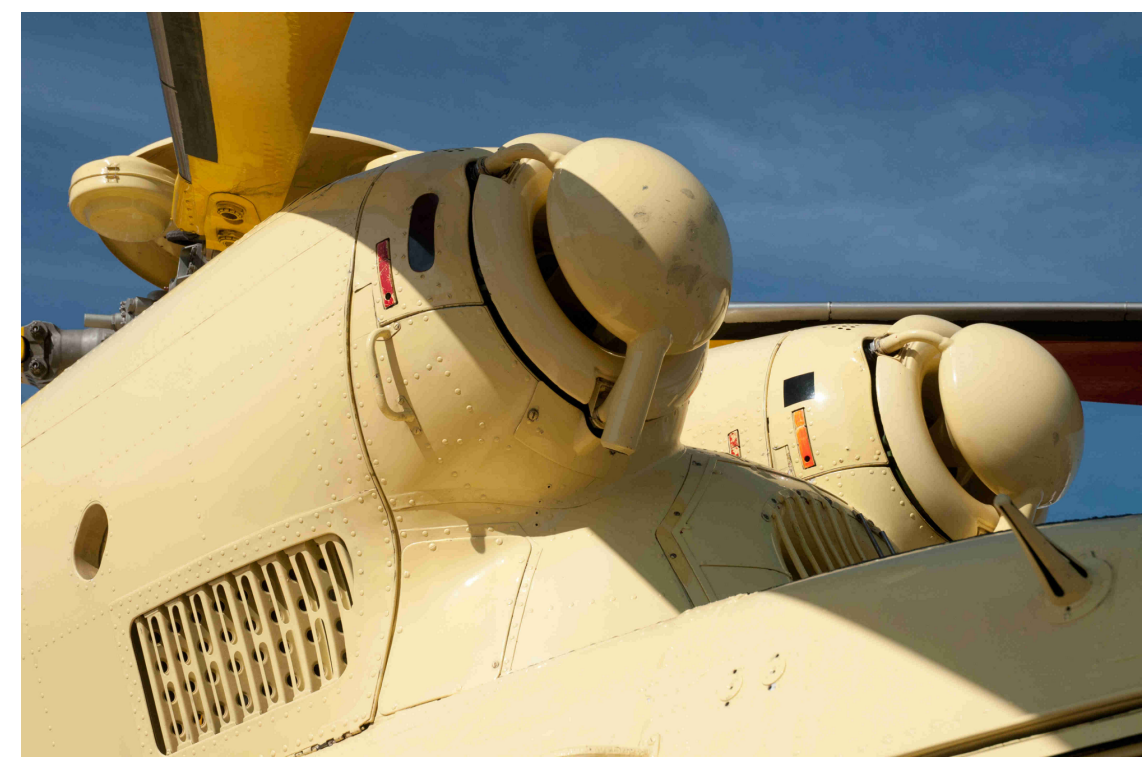

Figure 2: A typical external IPS, featured here on the PZL-Świdnik Kania.

\subsection{The Concept}

The self-cleaning IBF comprises of a cylindrical drum, whose circumferential wall is a pleated fabric filter. One end (base) of the drum is open, and attached to the turboshaft engine, while the other end is closed. The concept is illustrated in Figure 3. Air is drawn in through all pleats, such that 
the volume flow rate demanded by the engine is divided across the whole circumferential area of the drum. Particles are initially captured within the pleated filter medium, but begin to accumulate on the surface once a critical mass has been reached. The critical mass per unit area of a given dust collected by a given fibrous filter, is the point at which the pressure drop begins to rise more sharply, due to particles no longer being able to penetrate the medium. This occurs due to blinding, whereby larger particle block the pores; or by the formation of dendrites, whereby particles pile up on the surface of a fibre, reducing the pore size. For a planar filter medium the critical mass can be established through analysis of the pressure drop curve, which will exhibit a discontinuity at the point at which the filter cake - layer of surface particles - begins to grow. This is of significance in the present work: particles trapped within the filter are more difficult to expel than those lying on the surface, in spite of the fact that the internal clogging stage is associated with a slower pressure drop rise (see Rebaï et al. [8]. An ideal filter medium for the current application would have a low critical mass in order that as much mass as possible can be removed through cleaning.

The circumferential pleated filter rotates about the longitudinal axis of the drum, in order that cleaning may be enacted at each pleat once per drum rotation. To re-iterate, an investigation into the most appropriate method of filter cake removal is beyond the scope of the current work. However, one suggestion is to utilise compressor bleed air in a high jet efflux to effectively scrape-clean the pleat surface. In a similar application - so-called "PJAC" air cleaners for land-based military vehicles - the filtration momentarily stops as a back-flowing jet-pulse is activated to expel the accumulated particles. In such applications, the fibrous medium surface is pre-coated with a "nano-web" substrate - a $0.2-0.3 \mu \mathrm{m}$ cellulose fibre net - to capture 

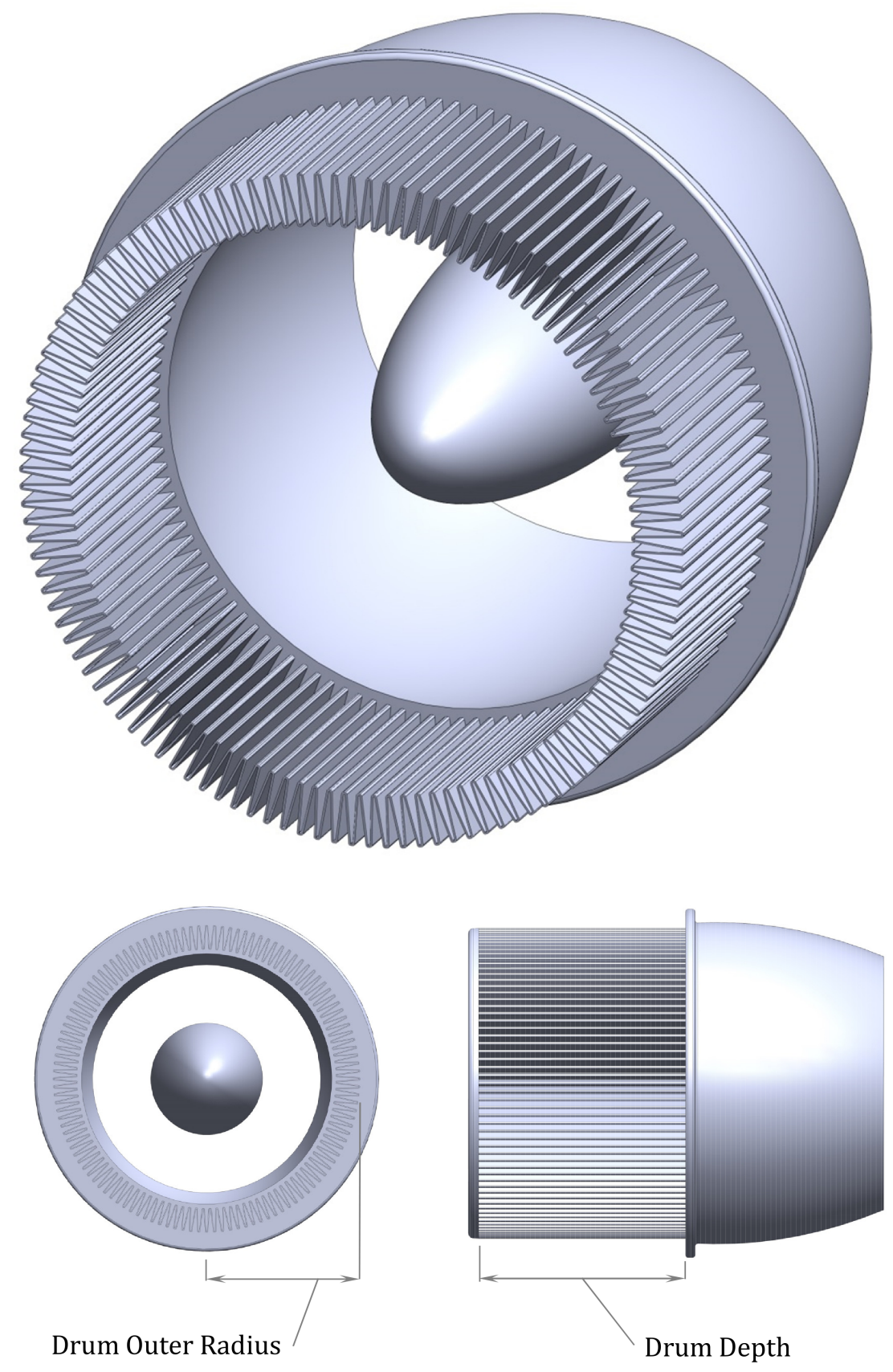

Figure 3: The self-cleaning drum attached to engine inlet, with cutaway view of pleats. 
particles as small as $1 \mu \mathrm{m}$ on the surface of the filter, thus aiding removal.

\subsection{The Model}

The ultimate objective is to predict the pressure drop as a function of time and ingested mass, but the task is made complicated by the number of dependent parameters involved. From the engine mass flow rate, to the dust type, to the internal structure of the filter; there are many variables to consider in forming a method. There are three main sources of pressure loss across the pleated interface: within the pleat channels, due to fluid shearing during contraction; within the medium, due to drag from the filter fibres; and within the cake, due to friction between the air and the accumulating particles. The first depends the pleat shape; the second depends on the structure of the chosen filter fabric; the third depends on the size distribution and compaction of the filter cake; and all three depend on the flow conditions. How these sources evolve is of great interest in the performance of a self-cleaning filter, since they are dependent on its design. Behind these dependent variables are independent design parameters that may dictate a number of variables. For example, the number of pleats one chooses to fit into the drum will have a bearing on the pleat shape and the rate of mass captured locally, which in turn affects the evolving pressure loss across each pleat.

To deal with such a plethora of dependent and independent variables, a multi-block system approach is adopted. The main components are depicted in Figure 4. The independent variable blocks are split into two groups: those set by the drum design (top left); and those dictated by the flow conditions (top right). These are separated further, with the structure depicted in Figure 4. 


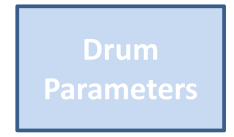

i. Rotation Speed

iii. Number of

Pleats

Filter Surface

Area

v. Pleat Aspect

Ratio

vi. Drum Length
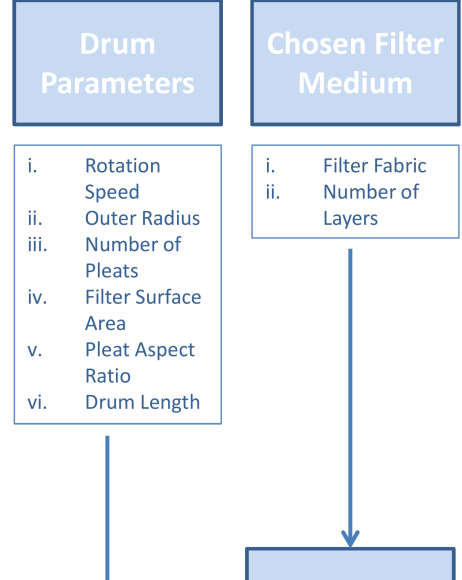

ii. Number of Layers

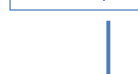

|

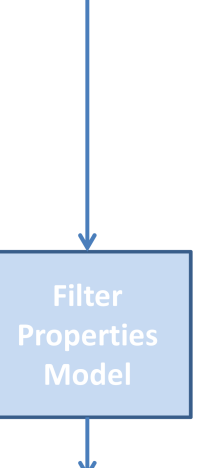

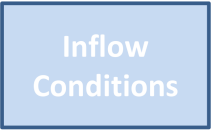

Engine Mass

Flow Rate
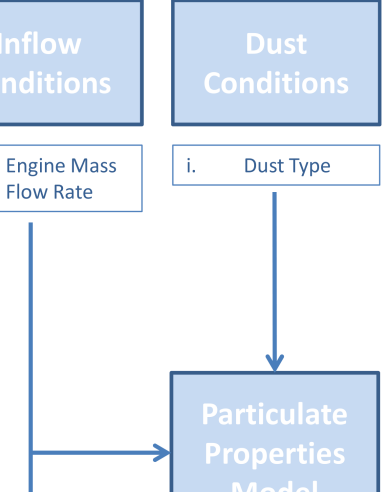

Atmospheric

Conditions

i. Dust Type

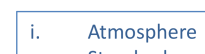

Standard Altitude

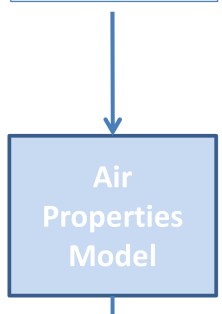

Mode
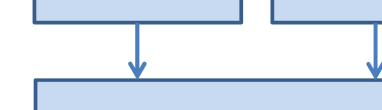

Cake Properties Model

Figure 4: Multi-block system for Self-cleaning IBF Performance Prediction model.

The flow parameters encompass all the external variables that are considered boundary conditions to the model. The inflow conditions include the engine mass flow rate, which in practice varies throughout the operation of the filter as the engine works at different speeds depending on demanding (for example, maximum RPM during takeoff at MTOW). The properties of the influent air depend on the altitude, and can be calculated by implementing the standard atmosphere values of temperature, pressure, density and viscosity into approximate empirical relationships based on the ideal gas law [17]. A dust sample is often represented by a particle size distribution (PSD), which breaks the dust sample into size bands according to their percentage by weight. Other useful properties of the dust sample may 
include an estimated volume shape coefficient, which is the relative radius of a spherical particle of the same volume; an average density; an uncompacted porosity; and a dynamic shape factor, which is the equivalent drag on a spherical particle of the same volume. Some known standard test dusts are available to use, such as SAE AC Fine Test, in order to compare similar devices. The dust concentration is also inputted here, given as a worst-casescenario single figure; in reality the concentration will fluctuate depending on the helicopter's flight mode, sometimes reducing to zero.

The drum design parameters are reduced to a set of six variables, which globally govern the remaining pleat dimensions that are required to solve the channel flow and numerically predict the flow across the pleated filter. The key dimensions of a half-pleat section are shown in Figure 5. (A half-pleat can be used in the numerical modelling the pleat flow thanks to symmetry, to save computational time). The number of pleats and drum circumference dictate the width of the pleat channel; the pleat depth is established by dividing the filter surface area by the number of pleats and choosing a pleat aspect ratio, defined as the channel exit width divided by the channel entry width (shown in Figure 5). The flow velocity approaching the pleat is the engine's volume flow rate divided by the total drum projected surface area. For example, for a drum of $0.4 \mathrm{~m}$ length, $0.3 \mathrm{~m}$ radius servicing an engine with a mass flow rate of $5 \mathrm{kgs}^{-1}$ at ICAO static sea level, the flow velocity into a pleat is approximately $6 \mathrm{~ms}^{-1}$.

The drum rotation speed determines the cycle time; when particles begin to collect on the surface of the filter, this is the key variable in the system. For a given particle collection rate, a faster rotating drum would result in a more frequent cleaning schedule at the expense of a shorter time window in which to remove all the collected particles. A faster-spinning drum may 
have additional implications on the flow, which would enter the channel with a tangential component; in the current work, the flow is considered to enter normal to the channel entry face. Incidentally, for the aforementioned example drum, a typical rotation speed of 12 RPM would introduce a tangential velocity component of approximately $0.4 \mathrm{~ms}^{-1}$, which is just $6 \%$ of the normal component.

The choice of filter medium will become essential in later permutations of the self-cleaning barrier filter. To encourage cleaning, a pre-coated filter, or a filter with a low particle retention and smooth surface finish is desired. The critical mass - as discussed above - at which the discontinuity in the pressure drop curve occurs is a significant value and is required in the model in order to establish when cleaning can be initiated. This is sometimes called the clogging point. In pleated form, a filter's clogging point may differ to that of the planar form owing to a variation in the incident angle of particle penetration; in the absence of real data for the medium in its pleated form, the planar clogging point is used. This is assumed to be provided for a given fabric and test dust, along with the slope of the pressure drop curve during the internal clogging stage. For the present study, known clogging data for a specific filter fabric used for similar flows in automobile applications are borrowed from Ref. [8]. The filter medium is a 2-ply fibrous mat, with an average fibre diameter in the first layer of $13 \mu \mathrm{m}$ and of $14 \mu \mathrm{m}$ in the second layer; the two layers contribute to a total thickness of $2.22 \mathrm{~mm}$. In its clean state, it has a permeability and porosity of $1.66 \mathrm{E}-10 \mathrm{~m}^{-2}$ and 0.9 respectively. The permeability is a measure of its ability to transmit fluid, and therefore reflects the filter's resistance.

The resistance gives rise to a pressure gradient across the filter medium, which is a function of the filtration velocity, $U_{F}$. The relationship between 
the filtration velocity, porous medium structure, and the corresponding pressure gradient across a porous medium has been studied since the work of Darcy in 1856 [18], and many improved or empirical relationships developed since are based on the same parameters. A review is given in Bear [19]. For flows of fibre Reynolds number $R e_{f}>10$, the pressure gradient is composed of a linear term and a quadratic term, as follows:

$$
-\nabla p_{F}=\mu_{g} C U_{F}+\frac{1}{2} \rho_{g} D U_{F}^{2}
$$

where $\mu_{g}$ is the kinematic viscosity, $\rho_{g}$ is the air density, and $C$ and $D$ are the viscous and inertial resistance coefficients. In absence of an experimentallyderived value, the widely used Ergun equation [19] can be used provided the fibre diameter, filter bulk density and filter material are known. The viscous and inertial resistance coefficients as given by Ergun for the filter medium are:

$$
\begin{gathered}
C=\frac{150\left(1-\epsilon_{F}\right)^{2}}{\epsilon_{F}^{3} d_{f}^{2}} \\
D=\frac{3.5\left(1-\epsilon_{F}\right)}{\epsilon_{F}^{3}}
\end{gathered}
$$

where $d_{f}$ is the fibre diameter and $\epsilon_{F}$ is the filter porosity (calculable from the bulk density and fibre density). It follows that as the filter medium collects particles, its porosity decreases. The transient filter porosity can be written as a function of collected mass:

$$
\epsilon_{F}\left(m_{p c}\right)=\epsilon_{F}(0)-\frac{\overline{\rho_{p}} m_{p c}}{Z_{F} A_{F}}
$$

where $\epsilon_{F}(0)$ denotes the initial porosity, $\overline{\rho_{p}}$ is the mean particle density, $m_{p c}$ is the collected particle mass, $Z_{F}$ is the filter thickness and $A_{F}$ is the filter surface area. The collected mass can be written as a function of the filter's efficiency for a particular particle size, as in Chapter 3.3.3 of Ref. [1], but 
in the current work the filter is assumed to be able to remove all particle sizes with $100 \%$ efficiency, as this represents a worst-case-scenario in terms of clogging.

In the model tree shown in Figure 4, the relevant properties of the filter are derived from the independent variables with separate pre-process module. To ascertain the collected particle mass introduced in Eq. 4, a similar "particulate properties" model is used, which takes as inputs the dust type and concentration, engine mass flow rate, and gas density to produce the mean particle diameters to represent the PSD, mass concentration, and particle mass flow rate. The arithmetic mean diameter of a fractional size distribution is:

$$
\bar{d}_{p}=\sum_{i=1}^{N_{p}} \bar{d}_{p, i} m_{i}^{+}
$$

where the index $i$ denotes a particle size group, $d_{p, i}$ denotes the representative particle size of that group, $m_{i}^{+}$is the fraction of the total mass comprised of the group, and $N_{p}$ is the number of particle. The mean particle size diameter for Arizona AC Fine test dust is $19 \mu \mathrm{m}$. The mass concentration is the particulate to gas mass ratio, and can be calculated by consideration of the volume fraction of each phase, expressed by their densities:

$$
c_{m}=\frac{1}{\rho_{g} / c_{v}+\left(1-\rho_{g} / \rho_{p}\right)}
$$

A proof is given in Ch. 3.2.1 of Ref. [1]. The particulate mass flow rate is then simply calculated from the engine mass flow rate. As the particulate collects on the filter surface, a new source of pressure loss is formed. There have been many studies dedicated to modelling filter cake growth, in which the pressure drop is predicted as a function of the filtration velocity and properties of the dust. One such model showing good agreement 
with experiment for AC Fine test dust is proposed by Endo et al. [20]. Unlike other models, it can be applied to very large particle Reynolds number flows $\left(\operatorname{Re}_{p} \leq 10^{4}\right)$ through cakes of a polydisperse distribution. The pressure gradient is described as:

$$
-\nabla p_{C}=\mu_{g} E U_{C}+\sqrt{\rho_{g} \mu_{g}} F U_{C}^{1.5}+\frac{1}{2} \rho_{g} G U_{C}^{2}
$$

where $U_{C}$ is the cake filtration velocity, and the coefficients $E, F$ and $G$ are functions of properties of the porous matrix, given by:

$$
\begin{gathered}
E=17.28 \frac{\left(1-\epsilon_{C}\right) v(\epsilon)}{\epsilon_{C}^{2}} \frac{\kappa}{d_{v g}^{2} \exp \left(4 \ln ^{2} \sigma_{g}\right)} \\
F=3.96 \frac{\left(1-\epsilon_{C}\right) v(\epsilon)}{\epsilon_{C}^{2.5}} \frac{\kappa}{d_{v g}^{1.5} \exp \left(\frac{27}{8} \ln ^{2} \sigma_{g}\right)} \\
G=\frac{\left(1-\epsilon_{C}\right) v(\epsilon)}{\epsilon_{C}^{3}} \frac{\kappa}{d_{v g} \exp \left(\frac{5}{2} \ln ^{2} \sigma_{g}\right)}
\end{gathered}
$$

where $\epsilon_{C}$ is the cake porosity, $\kappa$ is the dynamic shape factor, $d_{v g}$ and $\sigma_{g}$ are the geometric mean diameter and standard deviation respectively. The function $v(\epsilon)$ is called the void function, and is included to account for spread in data from experiments on the dust in question. In the absence of such data, an estimate from a work on the compressibility of fine fly ashes (of a similar geometric mean diameter to AC Fine test dust, but a narrower distribution) by Choi et al. [21] is given as:

$$
v(\epsilon)=165 \frac{\left(1-\epsilon_{C}\right)}{\epsilon_{C}^{2}}
$$

Prior work by the authors [12] established that under conditions typical of IBF, a cake composed of AC fine test dust with a thickness of the same order of magnitude as the filter thickness would not undergo significant compaction. Therefore the cake porosity is considered constant, at a value of 
0.65 (also borrowed from Ref. [8]). Since such relationships were developed from experiments on planar media, the current work assumes that the distribution of cake is homogeneous across the whole filter surface. In reality, the accumulation is likely to be non-uniform, and dependent on the local flow conditions and particle size [16]. This phenomenon is to be explored in future work.

The modules described above create the necessary variables to solve the flow in the pleat channels, which ultimately dictates how the whole system will perform. The principal task of the pleat flow properties model is to take the drum parameters, flow parameters, and filter parameters to generate a set of boundary conditions for use in a numerical solution to the NavierStokes equations, which in the present work is solved using commercial CFD software. In order to understand the consequences of varying the drum design parameters mentioned above upon which the pleat shape depends, the results of a previous parametric CFD study were utilised. In this study by Bojdo [1], a numerical simulation was conducted on a two-dimensional half-pleat section. The internal and external accumulation of particles and subsequent increase in flow resistance was enacted through prescription of a negative source (or sink) term to all cells in a region of the flow domain designated as porous. Such zones were drawn to reflect the half-pleat shape and a series of nine uniform thickness cake layers, as depicted in Figure 5. The prescription of the sink term to the relevant cells was achieved using Ansys' porous media model, whereby the user specifies a viscous term and an inertial term to reflect the pressure gradient across any cell lying in the porous zone. The sink terms were derived from the expressions given in Eq. 1 and Eq. 7. By incrementally changing the sink terms in accordance with Eq. 4 to reflect a decrease in filter porosity (internal clogging), or the 
mere presence of a filter cake, the effect of particle accumulation on the pleat could be simulated. For full details on the implementation of these and the computational procedure, see Ch. 4 of Ref. [1].

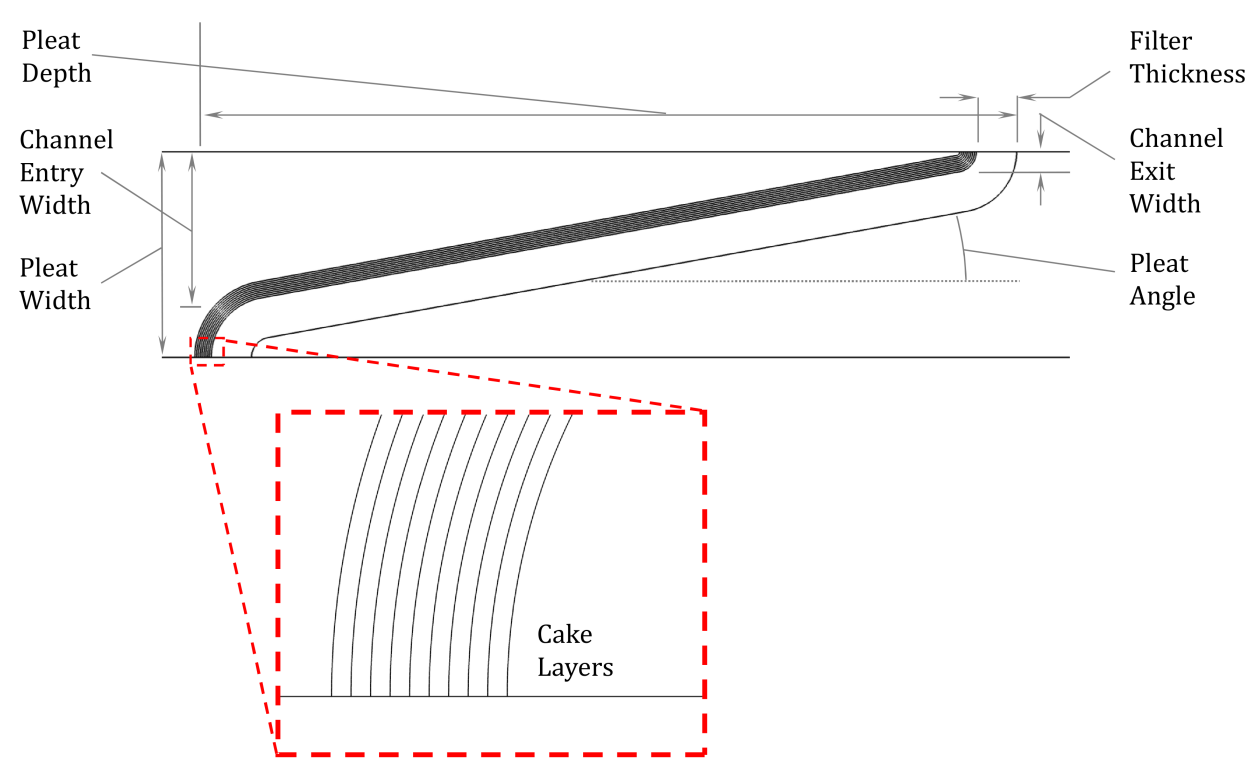

Figure 5: Half-pleat section with descriptive key dimensions labelled.

The CFD study yielded a large set of parametric data. A number of half-pleats of varying depth $(0.03 \mathrm{~m}-0.05 \mathrm{~m})$ and pleat angle $(1 \mathrm{deg}$. 10 deg.) were subjected to a range of inflow velocities $\left(2 \mathrm{~ms}^{-1}-12 \mathrm{~ms}^{-1}\right)$. For a particular half-pleat section, several runs were performed. During each run, the sink terms were updated to reflect the addition of particulate mass. Each mass increment was expressed a fraction of the absolute critical mass for each half-pleat, since each had a unique surface area hence capacity. At each interval ( $10 \%$ capacity; $20 \%$ capacity; and so on) the pressure drop was recorded. This continued when the half-pleat case reached capacity: a collected mass fraction of unity represents the point at which cake begins 
to form. This method thus afforded a consistent record of pressure drop versus mass collected for each of the pleat sections considered. The resulting four-parameter data set (pleat depth, pleat angle, inflow velocity and mass accumulated) generated was used to populate a set of data points that could be interpolated between for any pleat geometry within the range tested. This was hence incorporated into the model. It is noteworthy to say that result sets generated by other means, for example by experiment, could be imported as easily into the existing model provided all filter properties in the system are updated.

\section{Results \& Discussion}

The pleat flow properties model completes the set of models needed translate the flow and self-cleaning drum design parameters into useable variables. The final process uses these variables to produce a transient pressure drop as a function of mass collected by a single pleat. The pleat can be considered as one of a number of pleats arranged as shown in Figure 3. Each pleat is cleaned once per revolution at a fixed location on the circumference of the drum, such that no two pleats are rendered inactive due to cleaning at any one time. It follows that each pleat has spent a different length of time subjected to the oncoming particulate-laden flow, depending on its relative distance from the point of cleaning. It must be remembered that in this simplified model it is assumed that particles do not begin to accumulate on the filter surface and thus become "cleanable" until the filter's internal capacity has been reached. Figure 6 shows the transient pressure drop across one pleat of the drum. The key independent properties of the drum are summarised in Table 1 ; these are the default values when any one parameter is varied. All values are realistic and based on a self-cleaning IBF 
Table 1: Independent design and flow variables for self-cleaning drum simulation results.

\begin{tabular}{rrl}
\hline Height above Sea Level & 0 & $\mathrm{~m}$ \\
Engine Mass Flow Rate & 5.5 & $\mathrm{kgs}^{-1}$ \\
Dust Concentration & 2.5 & $\mathrm{gm}^{-3}$ \\
Dust Type & AC Fine & \\
& & \\
Drum Radius & 0.3 & $\mathrm{~m}$ \\
Drum Depth & 0.5 & $\mathrm{~m}$ \\
Number of Pleats & 120 & - \\
Total Filtration Area & 6 & $\mathrm{~m}^{2}$ \\
Pleat Channel Aspect Ratio & 0.2 & - \\
Drum Rotation Speed & 6 & $\mathrm{RPM}$ \\
\hline
\end{tabular}

solution for a medium-heavy lift rotorcraft engine.

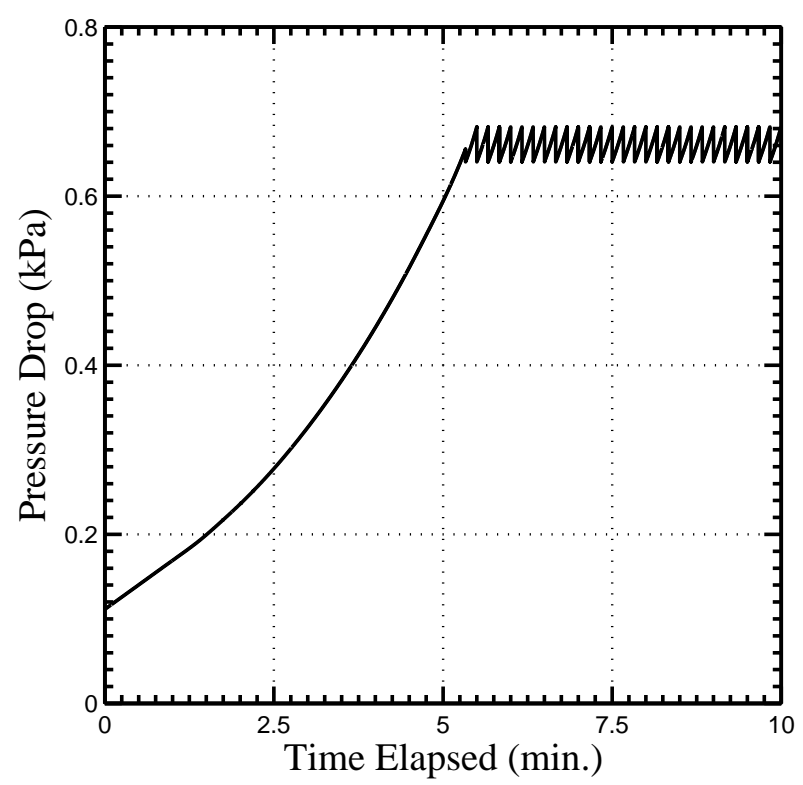

Figure 6: Pressure drop across a single pleat as a function of time elapsed since exposure to dust. 
The point at which the cyclic cleaning becomes effective is evidenced in Figure 6 by the onset of a fluctuating pressure drop signal. The period leading up to this point reflects the period of internal clogging during which particles cannot be removed. After this point, the pleat reaches a quasi steady state. Again, this is an ideal situation with uniform flow conditions (dust concentration, mass flow rate) and $100 \%$ cleaning efficiency; in reality the steady state signal may look different. For this parameter set, the pressure drop across the pleat fluctuates between 0.64 and $0.68 \mathrm{kPa}$, with a cleaning period equal to the period of rotation (10 seconds). From this, it is superfluous to announce that the extent to which the pressure drop can rise during one rotation depends on the speed of rotation. Recalling the definition of filter medium critical mass, the benefit of using a filter that holds less mass internally is that the cleaning cycles can begin sooner; combining this with a faster spinning drum allows the steady state pressure drop to be kept low. The limiting factor then becomes whether the pleat can be cleaned in a shorter space of time, since the cleaning period is the rotation period divided by the number of pleats.

A fluctuating pressure drop as exhibited in Figure 6 is, however low, not a desirable operational state with which to supply the engine, or helicopter pilot. Fortunately, by virtue of the fact that the cleaning cycles of each pleat in the drum are out of phase with one another, the mean pressure drop across the whole drum remains constant during this steady state period. This is illustrated in Figure 7, which expresses the total system pressure drop as a function of the total mass of particulate ingested by the engine intake. The steady state pressure drop for this set of parameters is $0.68 \mathrm{kPa}$, and begins only after approximately $3.4 \mathrm{~kg}$ of dust has been captured within the filter medium. This feature is the key benefit of the self-cleaning IBF over the 
other types of EAPS technology: maintaining a low intransient pressure loss while upholding the high separation efficiency of a fabric filter. Predicting this value is important, as this mass cannot be removed, and adds to the weight of the system, which must continue to be supported structurally. Incidentally, the clean filter pressure drop is approximately $0.1 \mathrm{kPa}$ in the given conditions.

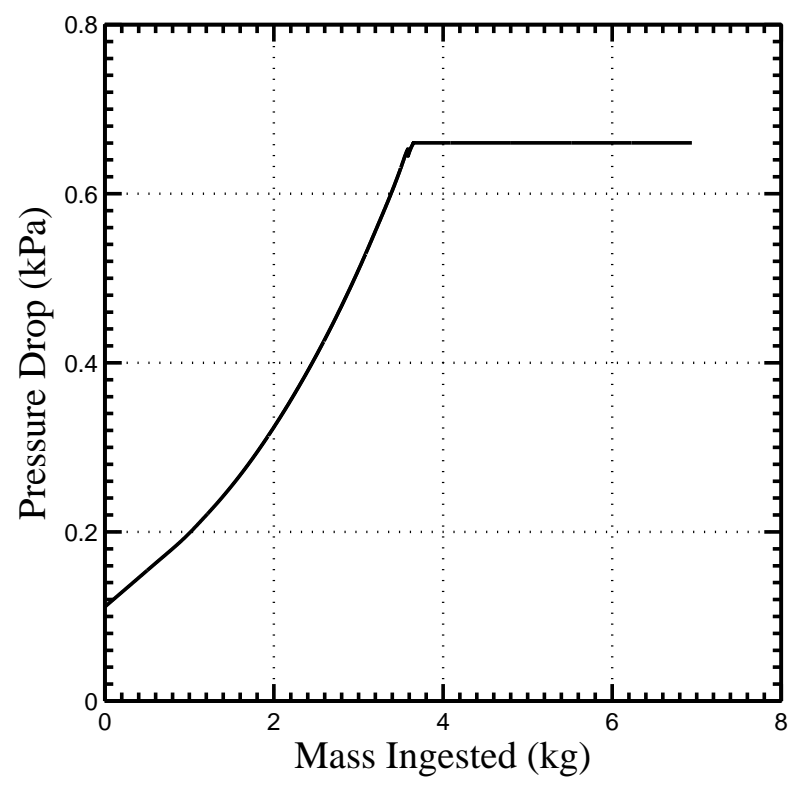

Figure 7: Pressure drop across whole system as a function of dust mass ingested.

Prior analysis of the result set used in this study (generated from CFD) indicates that the effect of deepening the pleat by adding more filtration area tends to reduce the pressure drop. Figure 8 illustrates this phenomenon for three different superficial flow velocities. Given that adding more "filter medium" essentially creates an additional source of pressure loss to the system, it stands to reason that the relationship exhibited in each curve in 
Figure 8 may resemble a parabola upon extrapolation. Unfortunately, the envelope of existing data set does not permit such an investigation. There may also be additional structural issues with an ever-increasing pleat depth.

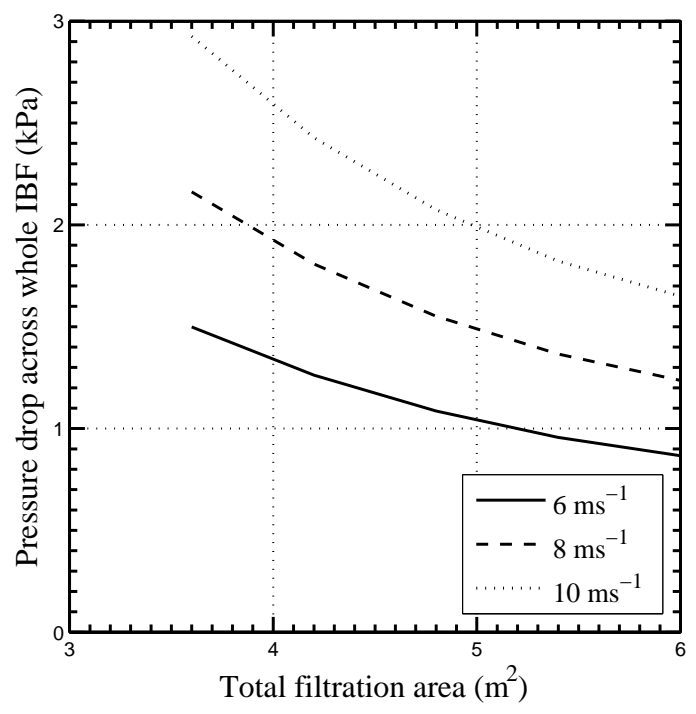

Figure 8: Effect of filtration area on steady state pressure drop.

The three curves shown in Figure 8 represent three superficial velocities (engine mass flow per unit projected area). Prior work by the authors [1] revealed that the throughput velocity is an influencing factor in the optimum pleat design for minimum pressure drop. Hence it is a good idea to manufacture the pleated filter with a design point velocity in mind. The superficial velocity is governed by three parameters shown in Table 1: engine mass flow rate; drum depth; and drum radius. Assuming the mass flow is fixed to reflect the demand at $100 \%$ engine speed and the drum radius is chosen fit the inlet profile, the superficial velocity is set by the drum depth. As evident in Figure 8, a lower superficial velocity is desired; however achieving this by extending the drum depth may be compromised by a lack of space in the 
snug intake plenum.

Evidence of an optimum design is evident, however, in the parabolicshaped curves of the pleat aspect ratio results (in which a ratio of 0 is a planar filter). Figure 9 demonstrates the key compromise in pleated filter design: an increasing aspect ratio means more "filter" per unit projected area, which translates to a smaller filtration velocity hence reduced loss across the medium. However the loss begins to increase again as the aspect ratio approaches unity due to the increased presence of shear stresses in the channel fluid. The optimum design point shows a slight tendency to a lower aspect ratio as inflow velocity increases. For this parameter set it is achieved when the channel exit width is approximately $40 \%$ of the channel entry width. A similar illustration of design optimisation is shown in Figure 10. While the pleat aspect ratio, filter thickness and pleat area remain fixed, the initial effect of increasing the number of pleats is a lowering of the pressure loss due to a lengthening of the pleat channels, but this gives way to an increase in pressure loss as the air-to-cloth ratio continues rising.

The drum rotation speed is the key novel design parameter in the present study. Figure 11 illustrates the benefit of increasing the drum RPM, which is to reduce the steady state pressure drop. The curves are hyperbolic, tending to a constant value once the rotation speed has breached 5 RPM (although if there were fewer pleats, this number would be larger and vice versa). The reason for this is obvious: a faster rotation speed means a shorter period of particle accumulation. However, this is an idealised situation in which the cleaning mechanism removes $100 \%$ of the surface cake despite having less time to do so (in this case approximately 0.1 seconds). Whether this is possible depends upon the method of extraction, a topic that is not covered in the current work. Similarly, there may be an effect of tangential inflow 


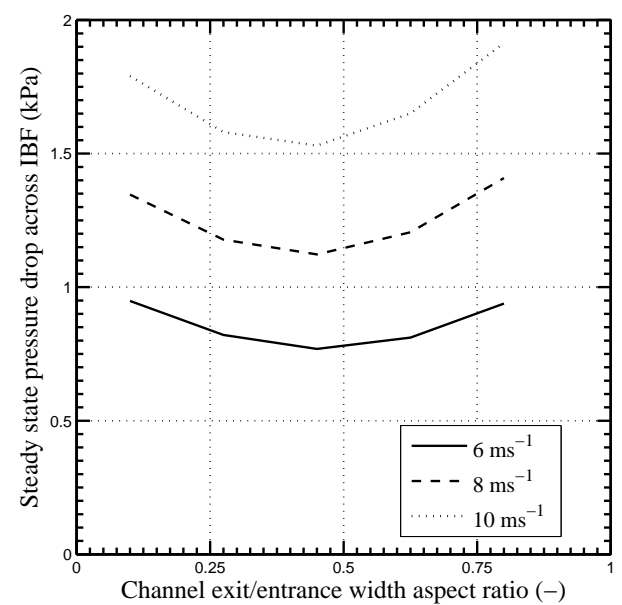

Figure 9: Effect of channel width aspect ratio on steady state pressure drop.

component on the pressure drop, as the filtration velocity through one half of a pleat would increase, akin to the effect of a reduced filtration area which has been shown to cause a larger loss of pressure in Figure 8. 


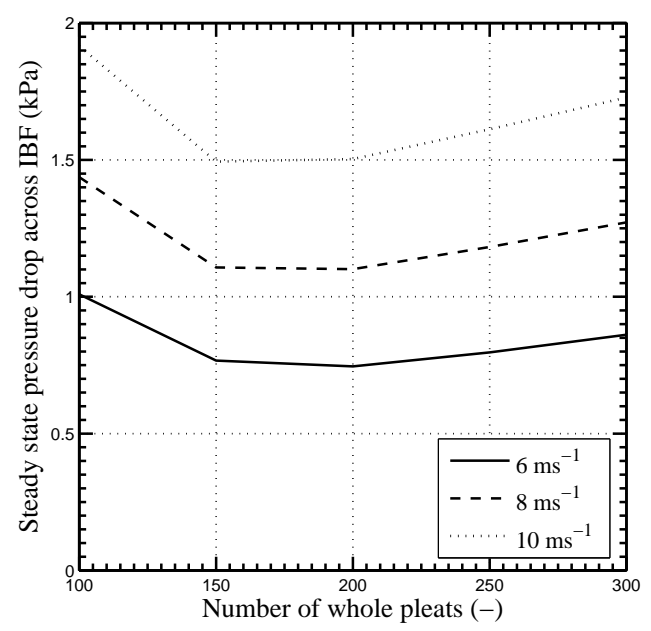

Figure 10: Effect of pleat number on steady state pressure drop.

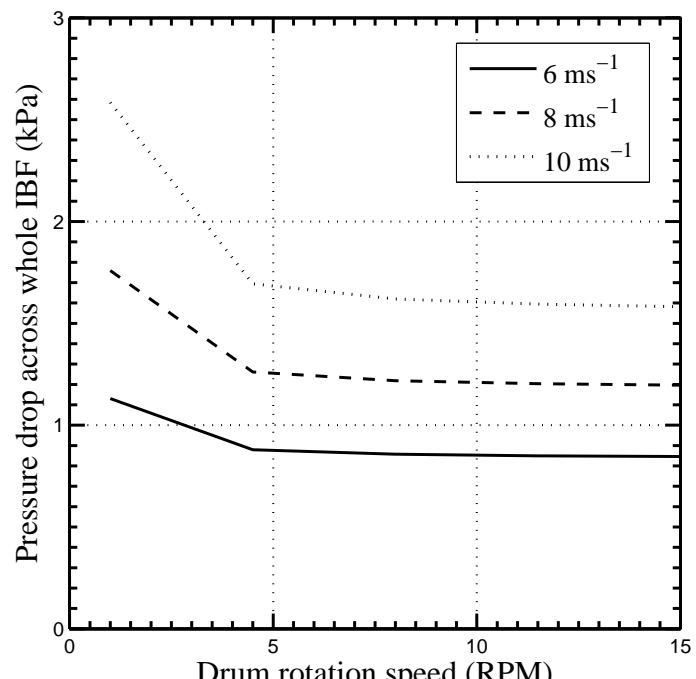

Figure 11: Effect of drum rotation speed on steady state pressure drop.

\section{Conclusions}

The work presented herein aimed to investigate the possibility of a novel self-cleaning inlet barrier filter for helicopter engines, from a design param- 
eter perspective. The main conclusions are as follows:

1. The self-cleaning barrier filter concept has been shown how it would achieve an invariant and low pressure loss while maintaining thr high separation performance of a fabric filter, giving it a distinct advantage over the other types of EAPS.

2. There are five sets of input boundary conditions: three are considered external and beyond control, which include the inflow conditions, dust type, and atmosphere standards; while the remaining two relate to the design of the self-cleaning IBF.

3. The IBF performance is judged by assessing the steady state pressure loss.

4. The choice of filter medium is paramount to the success of the selfcleaning concept. All planar filter media have a critical value of collected mass, beyond which particles will collect on the surface. Keeping this value low means cleaning can begin sooner, which keeps the steady state pressure loss low. However, since the critical mass is a function of the particulate and flow properties it is important to know the boundary conditions before choosing the medium.

5. The steady state pressure loss is reached when all pleats of the IBF have reached internal capacity and are being purged of surface dust cake once per drum rotation.

6. Under the parameter set studied in the present work, the drum IBF captures $3.4 \mathrm{~kg}$ of AC Fine dust within the filter medium before cleaning halts accumulation. This weight cannot be removed and would have to be supported by the structure of the self-cleaning IBF.

7. The second set of design parameters relate to the dimensions of the 
drum and pleats, of which there are six. The drum radius and drum depth control the throughput velocity, which is reduced by increasing one or both of these to the advantage of pressure loss, space permitting. The number of pleats and pleat aspect ratio can both be optimised to yield a minimum pressure loss; a similar trait is expected for the pleat area, but the envelope of the data used does not permit such conclusions.

8. The sixth parameter is the drum rotation speed. Under ideal conditions, in which a pleat is cleaned completely in a very short space of time, the steady state pressure drop tends to a minimum after the drum RPM has increased beyond a critical value.

Future work is intended to investigate the effect of a tangential flow component on the flow, which is the main phenomenological difference between this concept and existing pleated filters. Additionally, with validation from experimental results, the potential for optimising the self-cleaning drum can be exploited.

\section{References}

[1] N. Bojdo, Rotorcraft Engine Air Particle Separation. PhD Thesis, University of Manchester, 2012.

[2] X. Simon, S. Chazelet, D. Thomas, D. Bémer and R. Régnier, "Experimental study of pulse-jet cleaning of bag filters supported by rigid rings.", Powder Technology, vol. 172, no. 2, pp. 67-81, 2007.

[3] L-M. Lo, D-R. Chen, and D.Y.H. Pui, "Experimental study of pleated fabric cartridges in a pulse-jet cleaned dust collector.", Powder Technology, vol. 197, no. 3, pp. 141-149, 2010. 
[4] E.H. Tanabe, P.M. Barros, K.B. Rodrigues and M.L. Aguiar, "Experimental investigation of deposition and removal of particles during gas filtration with various fabric filters.", Separation and Purification Technology, vol. 80, no. 2, pp. 187-195, 2011.

[5] L-M. Lo, S-C. Hu, D-R. Chen and D.Y.H. Pui, "Numerical study of pleated fabric cartridges during pulse-jet cleaning.", Powder Technology, vol. 198, no. 1, pp. 75-81, 2010.

[6] B.H. Park, M-H. Lee, Y.M. Jo and S.B. Kim, "Influence of pleat geometry on filter cleaning in PTFE/glass composite filter.", Journal of the Air E Waste Management Association, vol. 62, no. 11, pp. 1257-1263, 2012.

[7] D-R. Chen, D.Y.H. Pui and B.Y.H. Liu, "Optimization of pleated filter design using a finite-element numerical method.", Journal of Aerosol Science, vol. 24, no. 1, pp. S39-S40, 1993.

[8] M. Rebaï, M. Prat, M. Meireles, P. Schmitz, and R. Baclet, "Clogging modeling in pleated filters for gas filtration," Chemical Engineering Research and Design, vol. 88, no. 4, pp. 476-486, 2010.

[9] N. Bojdo and A. Filippone, "Performance Prediction of Inlet Barrier Filters for Rotorcraft Engines," Journal of Aircraft, vol. 48, no. 6, pp. 19031912, 2011.

[10] A. Filippone and N. Bojdo, "Turboshaft engine air particle separation," Progress in Aerospace Sciences, vol. 46, no. 5-6, pp. 224-245, July 2010.

[11] N. Bojdo and A. Filippone, "Operational Performance Parameters of Engine Inlet Barrier Filtration Systems for Rotorcraft," The Aeronautical Journal, vol. 116, no. 1182, pp. 1-23, 2012. 
[12] N. Bojdo and A. Filippone, "Fabric Filter Performance of Helicopter Inlet Barrier Filters," Journal of the American Helicopter Society, vol. 57, no. 3, pp. 1-13, 2012.

[13] M. Taslim, A. Khanicheh, and S. Spring, "A numerical study of sand separation applicable to engine inlet particle separator systems," Journal of the American Helicopter Society, vol. 54, no. 4, pp. 042001-1-10, 2009.

[14] M. J. Scimone, D. Flesher, and S. Frey, "High Performance Barrier Filtration Systems," Proceedings of the 56th American Helicopter Society Annual Forum, Virginia Beach, VA, 2000.

[15] C. Ockier, H. Muller, L. Wolfgang, C. Kolb, and S. Benedikt, "Flight Testing an Engine Inlet Barrier Filter for the EC145," Proceedings of the 65th Annual American Helicopter Society Forum, Grapevine, Texas, 2009.

[16] S. Fotovati, S. A. Hosseini, H. Vahedi Tafreshi, and B. Pourdeyhimi, "Modeling instantaneous pressure drop of pleated thin filter media during dust loading," Chemical Engineering Science, vol. 66, no. 18, pp. 40364046, 2011.

[17] A. Filippone, Flight performance of fixed and rotary wing aircraft, Access Online via Elsevier, 2006.

[18] H. Darcy, "Les fontaines publiques de la ville de Dijon," V. Dalmont, 1856.

[19] J. Bear, "Dynamics of Fluids in Porous Media", Elsevier, 1st ed., 1972.

[20] Y. Endo, D-R. Chen, and D.Y.H. Pui, "Effects of particle polydispersity and shape factor during dust cake loading on air filters", Powder Technology, vol. 98, no. 3, pp. 241-249, 1998. 
[21] J-H. Choi, S-J. Ha, and H-J. Jang, "Compression properties of dust cake of fine fly ashes from a fluidized bed coal combustor on a ceramic filter", Powder technology, vol. 140, no. 1, pp. 106-115, 2004. 